\title{
Endohedral Metallofullerene as Molecular High Spin Qubit: Diverse Rabi Cycles in Gd2@C $\mathrm{C}_{79} \mathbf{N}$
}

Ziqi Hu ${ }^{1 \dagger}$, Bo-Wei Dong ${ }^{1 \dagger}$, Zheng Liu ${ }^{1}$, Jun-Jie Liu ${ }^{2}$, Jie Su${ }^{1}$, Changcheng $\mathrm{Yu}^{1}$, Jin Xiong ${ }^{1}$, DiEr Shi ${ }^{1}$, Yuanyuan Wang ${ }^{1}$, Bing-Wu Wang ${ }^{1}$, Arzhang Ardavan ${ }^{2}$, Zujin Shi ${ }^{*}$, Shang-Da Jiang $^{1 *}$ and Song Gao ${ }^{{ }^{*}}$

${ }^{1}$ National Laboratory for Molecular Sciences, State Key Laboratory of Rare Earth Materials Chemistry and Applications, College of Chemistry and Molecular Engineering, Peking University, Beijing 100871 P. R. China.

${ }^{2}$ CAESR, The Clarendon Laboratory, Department of Physics, University of Oxford, Oxford OX1 3PU, UK.

${ }^{\dagger}$ These authors contributed equally to this work.

\begin{abstract}
Anisotropic high spin qubit with long coherence time could scale the quantum system up. It has been proposed that the Grover's algorithm can be implemented in such systems. Dimetallic aza[80]fullerenes $\mathrm{M}_{2} @ \mathrm{C}_{79} \mathrm{~N}$ (M=Y or Gd) possess unpaired electron located between two metal ions, offering an opportunity to manipulate spin(s) protected in the cage for quantum information processing. Herein, we report the crystallographic determination of $\mathrm{Gd}_{2} @ \mathrm{C}_{79} \mathrm{~N}$ for the first time. This molecular magnet with collective high spin ground state $(S=15 / 2)$ generated by strong magnetic coupling $\left(J_{G d-R a d}=350 \pm 20 \mathrm{~cm}^{-1}\right)$ have been unambiguously validated by magnetic susceptibility experiments. $\mathrm{Gd}_{2} @ \mathrm{C}_{79} \mathrm{~N}$ behaves quantum coherence and diverse Rabi cycles, allowing arbitrary superposition state manipulation between each adjacent level. The phase memory time reaches $5 \mu \mathrm{s}$ at $5 \mathrm{~K}$ by dynamic decoupling. This molecule fulfills the requirement of Grover's searching algorithm proposed by Leuenberger and Loss.
\end{abstract}

\section{INTRODUCTION}

Endohedral metallofullerenes (EMFs) provide a paradigm to investigate the behaviors of encapsulated atom(s) or spin(s) in the confined space ${ }^{1}$. Molecular nanomagnets with slow magnetic relaxation can be synthesized by entrapping paramagnetic ion(s) like $\mathrm{Dy}^{3+}$ and $\mathrm{Tb}^{3+}$ into fullerene cage, showing potential application in information storage $e^{2-5}$. On the other hand, stable radical may be generated when there is an unpaired electron $(S=1 / 2)$ stemming either from encapsulated cluster or azafullerene cage in the cases of $\mathrm{Sc}_{3} \mathrm{C}_{2} @ \mathrm{C}_{80}{ }^{6}$ and $\mathrm{Y}_{2} @ \mathrm{C}_{79} \mathrm{~N}^{7}$, respectively. Tunable magnetic property can be further realized in these systems through chemical functionalization and selfassembly, affording a feasible option to design spintronic devices for understanding of quantum behaviors and hence manipulation of electron $\operatorname{spin}^{8-12}$.

Quantum computation (QC) could outperform classical approaches in cryptography and database searching ${ }^{13-15}$, which requires long-lived quantum coherence and manipulation of arbitrary superposition quantum states. The Grover's algorithm is to search an element in a chaotic list based on probability amplification method. It is possible to implement the Grover's algorithm into multilevel systems so as to avoid the entangled quantum gates ${ }^{16}$. Magnetic molecules are the good candidates for this sort of multilevel system, since molecular spins are easily tunable by rational 
design. Recently, Wernsdorfer have realized the Grover's algorithm on the nuclear spin of a single molecule magnet ${ }^{17}$. Leuenberger and Loss have proposed implementing Grover's searching algorithm in a molecular magnet with well-defined electron high spin ground state ${ }^{18}$. This proposal requires that the spin multiplet exhibiting anisotropy which results in non-equidistant energy levels, and most importantly, the quantum phase memory time must be long enough to implement the algorithm through unitary operations. However, among the various anisotropic molecular magnets with large spins, most are not suitable for implementing Grover's algorithm. This is principally because the quantum coherence can easily be destroyed by coupling to the environment in anisotropic high spin systems. Recently, Coronado and Barbara's groups show that single ion Gdbased qubits are outstanding candidates for $\mathrm{QC}^{19-20}$. In contrast to the previously reported molecular magnets of collective ground high spin states ${ }^{21-22}$, the small crystal field splitting of $4 \mathrm{f}^{7}$ electronic configuration paves the way to the quantum manipulations over a set of coherent transitions. These single ion qubits fulfill the requirements of Grover's algorithm and could even addressing multiqubits within a single ion, providing an alternative method to scale the quantum system up.

To search for qubit with larger spin and prolonged quantum coherence, Gd-containing EMF may be a rational choice with high-spin $\mathrm{Gd}^{3+}$ ion(s) protected in carbon cage. Fu et al. ${ }^{23}$ have synthesized a highly stable endohedral azafullerene $\mathrm{Gd}_{2} @ \mathrm{C}_{79} \mathrm{~N}$. W-band cw-EPR result indicated a high spin state of $S=15 / 2$, which was confirmed by theoretical investigation revealing strong coupling among three spin carriers in the molecule ${ }^{24}$. The anisotropic high-spin nature of $\mathrm{Gd}_{2} @ \mathrm{C}_{79} \mathrm{~N}$ offers us possibility to investigate the spin qubit property and further realize quantum control on molecular ensemble, which is rarely reported on EMFs. Besides, the structure of $\mathrm{Gd}_{2} @ \mathrm{C}_{79} \mathrm{~N}$ has not been fully unveiled. The structure-related symmetry analysis also is indispensable for determination of its multi-energy levels and understanding of superposition quantum states.

Herein, we report for the first time the crystallographic characterization, magnetic susceptibility, quantum coherence and diverse Rabi cycles of $\mathrm{Gd}_{2} @ \mathrm{C}_{79} \mathrm{~N}$ with well-defined high spin ground state $S=15 / 2$. The Gd-electron coupling constant is determined to be $350 \pm 20 \mathrm{~cm}^{-1}$, leading to large magnetic moment of $14.7 \mu_{B}$. The crystal structure reveals $C_{s}$ or lower symmetry of the whole molecule. The N-substitution of $I_{h}$-C 80 cage affords an unpaired electron $\left(S_{\text {ele }}=1 / 2\right)$ located between metal ions, which ferromagnetically couples with the two $\mathrm{Gd}^{3+}$ ions $\left(S_{G d}=7 / 2\right)$. The quantum phase memory time of the high spin state reaches $5 \mu$ s at $5 \mathrm{~K}$, and the diverse Rabi cycles allows arbitrary superposition state manipulation between each adjacent level. To the best of our knowledge, this molecule possesses the largest ground state that behaves multiple Rabi cycles. 


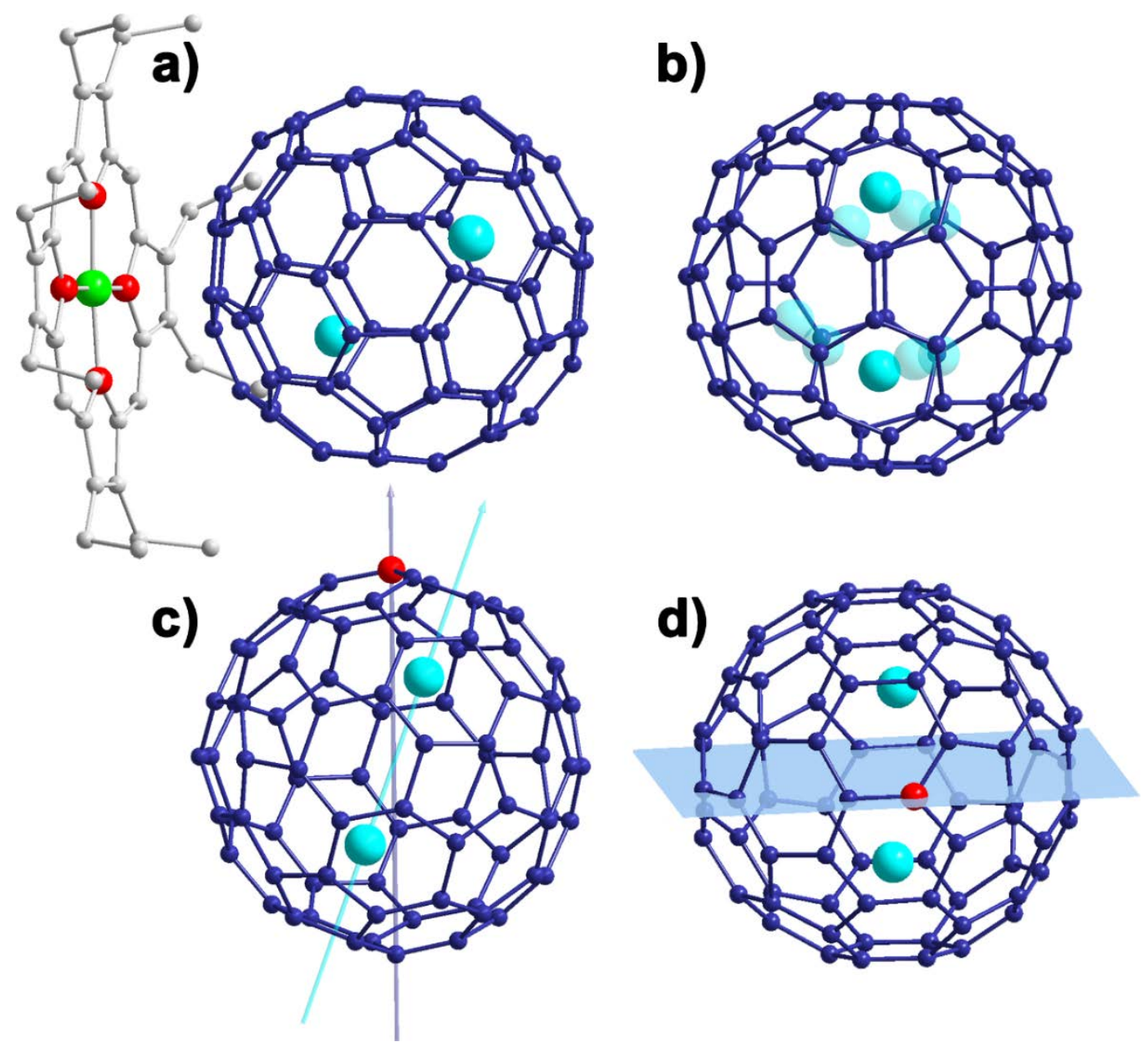

Figure 1 | Molecular structure of $\mathbf{G d}_{2} @ \mathbf{C}_{79} \mathbf{N}$. a) The crystal structure of $\mathrm{Gd}_{2} @ \mathrm{C}_{79} \mathrm{~N} \cdot \mathrm{Ni}{ }^{\mathrm{II}}(\mathrm{OEP})$ showing the unsymmetrical cage orientation with 0.50 fractional occupancy. The symmetry-related orientation generated by the crystallographic mirror plane has 0.50 occupancy. Only the major Gd sites are depicted. The solvent molecules and hydrogen atoms are omitted for clarity. b) The $\mathrm{Gd}^{3+}$ ion disorder plot with all minor Gd sites (cyan in part transparent) along with major sites (cyan), except for those generated by the crystallographic mirror plane. c) The non-collineation of the azafullerene 3-fold axis (blue) in case of a 666 substitution and the Gd-Gd line (cyan). d) An example of the $C_{s}$ symmetry when the 566 junction is substituted (the light blue plane indicates the mirror).

\section{RESULTS AND DISCUSSION}

Crystal structure and symmetry analysis. The synthesis, isolation and spectroscopic characterizations of $\mathrm{Gd}_{2} @ \mathrm{C}_{79} \mathrm{~N}$ are described in supplementary information. With two $\mathrm{Gd}^{3+}$ ions encapsulated in an azafullerene, $\mathrm{Gd}_{2} @ \mathrm{C}_{79} \mathrm{~N}$ is unambiguously crystallographically characterized. Co-crystallization of $\mathrm{Gd}_{2} @ \mathrm{C}_{79} \mathrm{~N}$ and $\left[\mathrm{Ni}^{\mathrm{II}}(\mathrm{OEP})\right]$ (OEP=octaethylporphyrin) by slow diffusion method yielded proper single crystals for X-ray structure determination for the first time. The molecules crystallize in $C_{2 / m}$ space group. Figure 1a shows the crystal structure of $\mathrm{Gd}_{2} @ \mathrm{C}_{79} \mathrm{~N}$ together with an adjacent [ $\left.\mathrm{Ni}^{\mathrm{II}}(\mathrm{OEP})\right]$ moiety. The 80 -atom cage possesses two orientations both with 0.50 fractional occupancy generated by the crystallographic mirror plane. Disordered locations 
of the Gd ions inside the cage can be observed (Figure 1b). The major Gd sites (i.e., Gd3 and Gd5A), with occupancy of $0.207(8)$ and 0.210 (3) versus the cage occupancy of 0.50 (Figure S1.3), resides under hexagonal rings with a $3.835 \AA$ Gd-Gd separation, featuring a quasi- $\eta^{6}$ coordination fashion similar to the situation in $\mathrm{Tb}_{2} @ \mathrm{C}_{79} \mathrm{~N}^{7}$. The two Gd-to-ring-centroid distances are $2.022 \AA$ for Gd3 and $1.928 \AA$ for Gd5A, respectively. The distances of Gd5A major site to the nearest carbons range from 2.339 to $2.435 \AA$, which is comparable to those in $\mathrm{Gd}_{3} \mathrm{~N} @ I_{h}-\mathrm{C}_{80}(2.344-2.439 \AA)^{25}$. In terms of Gd3 major site, however, it deviates slightly from the position right beneath the hexagonal ring, resulting in a larger Gd-C distances range (2.246-2.697 $\AA$ ).

The idealized $\mathrm{C}_{80}$ cage is of $I_{h}$ symmetry, whereas an $\mathrm{N}$ substitution of $\mathrm{C}$ atom reduces the symmetry to $C_{3 v}$ (666 junction) or $C_{s}$ (566 junction). However, it is not possible to identify the location of $\mathrm{N}$ atom on the cage from the crystal structure point of view due to the similar electron density of $\mathrm{N}$ and $\mathrm{C}$. Previous theoretical investigation suggested that the 566 junction substitution favors a lower energy ${ }^{7,23}$. One can infer that, by encapsulating the two $\mathrm{Gd}^{3+}$ ions in the cage, the highest possible symmetry is $C_{3 v}$ in the 666 substitution case when the two metal ions are collinear with the 3-fold axis. While the crystal structure reveals that the closest 3-fold axis is far apart from the Gd-Gd line (Figure 1c). This illustrates that the highest possible symmetry of $\mathrm{Gd}_{2} @ \mathrm{C}_{79} \mathrm{~N}$ is of $C_{s}$ point group. For instance, Figure $1 \mathrm{~d}$ shows an example of $C_{s}$ symmetry when carbon atom at 566 junction is substituted.
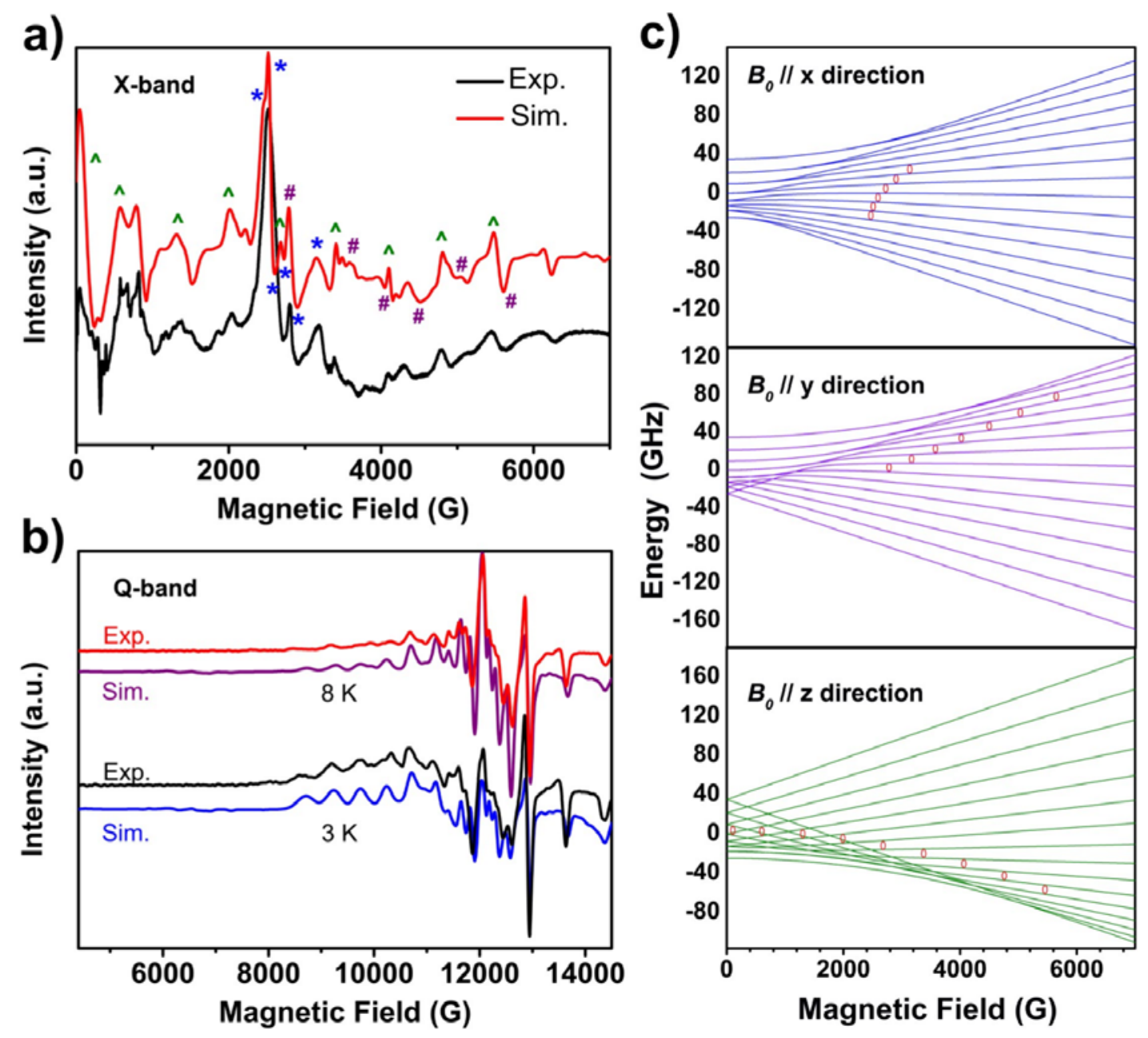

Figure $2 \mid \mathrm{X}-(20 \mathrm{~K}) / \mathrm{Q}-(3 \mathrm{~K}$ and $8 \mathrm{~K})$ band EPR spectra and energy levels of $\mathrm{Gd}_{2} @ \mathrm{C}_{79} \mathrm{~N}$ in 
frozen toluene solution. a) The X-band cw-EPR spectrum (black line) recorded at $20 \mathrm{~K}$. The simulation line is plotted in red. The best simulation is achieved with the parameters $B_{2}^{0}=0.953(6)$ $\mathrm{GHz}, B_{2}^{2}=0.288(6) \mathrm{GHz}, B_{4}^{0}=6.0(6) \times 10^{-5} \mathrm{GHz}$ and $g_{\text {iso }}=1.99$. The symbols “*”, “\#” and “^” denote the transitions along the magnetic principal axes $x, y$, and $z$ directions, respectively. b) The derivative of the echo detected field swept Q-band pulse-EPR spectra at $3 \mathrm{~K}$ (black) and $8 \mathrm{~K}$ (red) along with simulation results (blue and purple for 3 and $8 \mathrm{~K}$, respectively) depicted below using the same parameters as a. c) The 16 -fold energy levels of the $S=15 / 2$ multiplet as a function of the magnetic field $\mathrm{B}_{0}$ along the $x$ (blue), $y$ (violet) and $z$ (green) directions. The red circles indicate the positions of the observed Rabi cycles.

Magnetic properties. The $\mathrm{N}$-substitution affords an unpaired electron $\left(S_{\mathrm{rad}}=1 / 2\right)$, which unavoidably couples with the two $\mathrm{Gd}^{3+}$ ions $\left(S_{G d}=7 / 2\right)$. Previous studies demonstrated that such complexes exhibit strong ferromagnetic coupling between the three spin carriers ${ }^{4,23-24}$. To elucidate this ferromagnetic interaction between the spin centers and understand the electronic structure of this system, magnetic property measurements were performed on magnetometry. The field dependent magnetization $(\mathrm{MH})$ was measured at $2 \mathrm{~K}, 5 \mathrm{~K}, 8 \mathrm{~K}$ and $10 \mathrm{~K}$ in the range of 0 to $5 \mathrm{~T}$. It is clear that the $M H$ curves fulfill Brillouin function with a temperature dependence (Figure S2.1). At lowest temperature of $2 \mathrm{~K}$, the saturation magnetization is determined to be $14.7 \mu_{\mathrm{B}}$, in consistence with the $S=15 / 2$ ground state. The more informative experiment is the magnetic susceptibility vs temperature measurement at $1 \mathrm{~T}, 3 \mathrm{~T}$ and $5 \mathrm{~T}$ in the range of 2 to $300 \mathrm{~K}$. The $\chi_{m} T$ values at room temperature are determined to be 27.1 to $28.2 \mathrm{emu} \cdot \mathrm{K} / \mathrm{mol}$ for the three fields, which is far larger than three independent spin carriers expected value $16.125 \mathrm{emu} \cdot \mathrm{K} / \mathrm{mol}$ (one $S_{\mathrm{rad}}=1 / 2$, $g_{\mathrm{rad}}=2$ and two $\left.S_{G d}=7 / 2, g_{\mathrm{Gd}}=2\right)$, but close to the spin multiplet of $15 / 2(31.875 \mathrm{emu} \cdot \mathrm{K} / \mathrm{mol})$ or $13 / 2(24.375 \mathrm{emu} \cdot \mathrm{K} / \mathrm{mol})$ value. This is a strong indication that the magnetic coupling between the radical and $\mathrm{Gd}^{3+}$ could be very strong. The $\chi_{m} T-T$ curves (Figure S2.2) can be simulated by the Hamiltonian,

$$
\begin{aligned}
& \widehat{H}=g_{R a d} \mu_{B} \boldsymbol{B} \widehat{\boldsymbol{S}}_{\text {rad }}+g_{G d 1} \mu_{B} \boldsymbol{B} \widehat{\boldsymbol{S}}_{G d 1}+g_{G d 2} \mu_{B} \boldsymbol{B} \widehat{\boldsymbol{S}}_{G d 2}+J_{G d-G d} \hat{S}_{G d 1} \hat{S}_{G d 2} \\
& +J_{G d-R a d} \hat{S}_{G d 1} \hat{S}_{R a d}+J_{G d-R a d} \hat{S}_{G d 2} \hat{S}_{R a d},
\end{aligned}
$$

with the parameters $g_{R a d}=2.003$ and $g_{G d 1}=g_{G d 2}=1.98, J_{G d-G d}<1 \mathrm{~cm}^{-1}, J_{G d-R a d}=-350 \pm$

$20 \mathrm{~cm}^{-1}$. Using these parameters, the temperature dependent $M H$ curves can also be well-reproduced (Figure S2.1).

This abnormal strong coupling is attributed to the charge transfer from $\mathrm{N}$ atom to $\mathrm{Gd}^{3+}$ ion as predicted by previous DFT calculation ${ }^{23-24}$, illustrating the large degree of $5 d$ characterized spin density on the $\mathrm{Gd}_{2}$ cluster through NBO analysis. This indicates that there exists direct exchange between $\mathrm{Gd}^{3+}$ and the $\mathrm{N}$ centered radical on the cage. More importantly, the charge transfer hypothesis was also confirmed by the cw-EPR research on a similar molecule $\mathrm{Y}_{2} @ \mathrm{C}_{79} \mathrm{~N}^{7}$ with observation of yttrium involved large hyperfine coupling $(81.23 \mathrm{G})$ and reduced $g$ factor $(g=$ 1.9740).

We failed to determine the exact value of Gd-Gd coupling constant due to weak overlap of two $\mathrm{Gd}^{3+} 4 \mathrm{f}$ orbitals and hence relatively weak Gd-Gd coupling compared to Gd-radical interaction. The two $\mathrm{Gd}^{3+}$ ions are separated with $3.835 \AA$, which results in a small dipole-dipole interaction $(0.03$ $\mathrm{cm}^{-1}$ in $J_{d i p} S_{G d 1} S_{G d 2}$ convention). Interestingly, in our research, the spin carriers are still well coupled 
at room temperature, featuring a single peak of $g=1.98$ in cw-EPR spectrum instead of showing the signal of radical $(g=2.003)$ as in the case of $\mathrm{Gd@} \mathrm{C}_{82}{ }^{26}$. This implies that the strong Gd-radical ferromagnetic coupling dominates the interaction up to room temperature.

According to our simulation results, the $\chi_{m} T-T$ curves measured at different magnetic field (1 $\mathrm{T}, 3 \mathrm{~T}$ and $5 \mathrm{~T}$ ) can be well reproduced with the same $J$ between Gd1-radical and Gd2-radical. Besides, the spin density on the $\mathrm{C}_{79} \mathrm{~N}$ cage is localized on the $\mathrm{N}$ atom ${ }^{24}$. Consequently, it is highly possible that the $\mathrm{N}$ atom locates between two Gd centers, facilitating efficient charge transfer in this system. Furthermore, the previous studies have demonstrated that 566 substitution is more energetically favorable than 666 substitution $^{23-24}$. With the above discussions, we can speculate the possible location of $\mathrm{N}$ atom on the fullerene cage: on the plane shown in Figure 1d, and it should reside at a 566 junction position.

Energy spectrum. For $\mathrm{Gd}^{3+}$ ion, the orbital angular momentum of the ground multiplet is completely quenched due to the half-filled f-shell electron configuration. However, the mixing of the excited multiplet with the ground one as well as the local symmetry broken can still result in the slight crystal field splitting of the $\mathrm{Gd}^{3+}$. As the isolated ground state maintains at $20 \mathrm{~K}$ owing to large energy gap $\left(174 \mathrm{~cm}^{-1}\right)$ between the ground state $(S=15 / 2)$ and the first excited state $(S=13 / 2)$, it is reasonable to simplify the system to a giant spin model which considers only the ground spin multiplet $S=15 / 2$. With respect to the molecular symmetry analysis discussed above, the $C_{3 v}$ possibility can be fully ruled out. In this regard, there is no need to take $B_{4}^{3}$ term into consideration. Therefore, Hamiltonian of the system with $C_{s}$ or $C_{1}$ molecular symmetry is written as

$$
\widehat{H}=B_{2}^{0} \hat{S}_{z}^{2}+B_{2}^{2} \frac{\hat{S}_{+}^{2}+\hat{S}_{-}^{2}}{2}+B_{4}^{0}\left[35 \hat{S}_{z}^{4}-\hat{S}_{Z}^{2}(30 \tilde{s}-25)\right]+g_{i s o} \mu_{B} \boldsymbol{B} \widehat{\boldsymbol{S}},
$$

where the first two terms describe the second order zero-field splitting (ZFS) for rhombic symmetry, the third term is fourth order axial operator term, the last term is the Zeeman effect, and the symbol $\tilde{s}$ denotes $S(S+1)$. As no hyperfine interaction evidence between the electron and the nuclear spin $\left({ }^{14} \mathrm{~N},{ }^{155} \mathrm{Gd}\right.$ and $\left.{ }^{157} \mathrm{Gd}\right)$ is observed, the hyperfine and nuclear quadrupole terms are not included in the Hamiltonian and not discussed in the following. The EPR spectra at X- and Q-band (Figure 2) in frozen solution can be well reproduced by the parameters $B_{2}^{0}=0.953(6) \mathrm{GHz}, B_{2}^{2}=0.288(6)$ $\mathrm{GHz}, B_{4}^{0}=6.0(6) \times 10^{-5} \mathrm{GHz}$ and $g_{\text {iso }}=1.99$. To reproduce the inhomogeneous broadening, which originates from the $\mathrm{Gd}^{3+}$ ions disorder nature and various possible $\mathrm{N}$-atom locations, a ZFS strain $\left(\operatorname{Str}_{B 20}=0.060 \mathrm{GHz}\right.$ and $\left.\operatorname{Str}_{B 22}=0.045 \mathrm{GHz}\right)$ is applied in the spectra simulation. From the EPR spectra, we conclude that the present molecule offers a 16-fold level structure and all the two-level subspaces are addressable due to the well-defined anisotropy. 

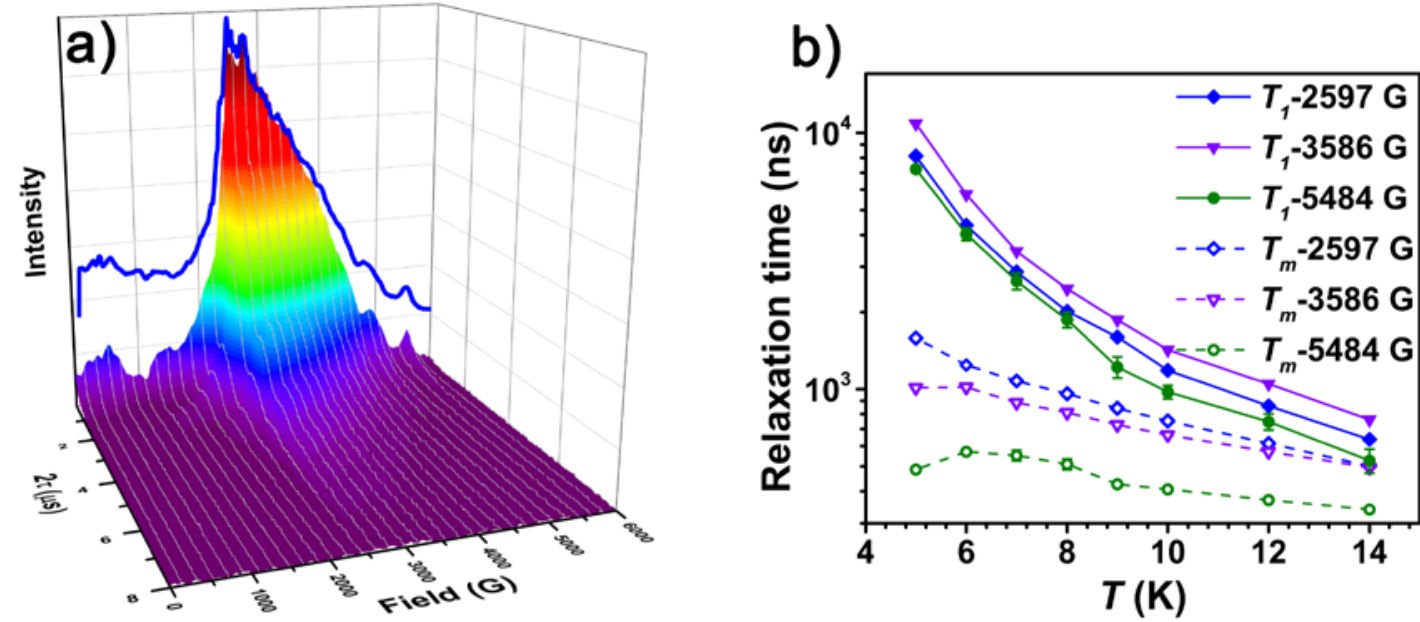

Figure 3 | The quantum coherence properties. a) The 3-dimensional plot of the Hahn echo decay in the field range of 2 to $6000 \mathrm{G}$ at $6 \mathrm{~K}$. The blue line indicates the echo-detected field-sweep spectrum. b) The temperature dependence of the spin-lattice relaxation time ( $T_{1}$ in solid symbols) and the quantum phase memory time ( $T_{\mathrm{m}}$ in open symbols). The error bars denote the standard error in $T_{1}$ and $T_{m}$, some of which are within the size of the symbols. The blue, violet, and green points are measured at the fields of 2597, 3586 and $5484 \mathrm{G}$, respectively. They correspond to the transitions of $\left|-\frac{3}{2}\right\rangle \leftrightarrow\left|-\frac{1}{2}\right\rangle$ (x direction), $\left|+\frac{1}{2}\right\rangle \leftrightarrow\left|+\frac{3}{2}\right\rangle$ (y direction) and $\left|-\frac{7}{2}\right\rangle \leftrightarrow\left|-\frac{5}{2}\right\rangle$ (z direction). The quantum numbers here are attributed by the high-field approximation.

Quantum coherence behavior. We have investigated the quantum coherence of the molecule in its $S=15 / 2$ ground spin multiplet by X-band pulsed EPR experiments. Using a Hahn echo sequence ( $\pi / 2-\tau-\pi$ - $\tau$-echo with $\pi / 2$ - and $\pi$-pulses of 50 ns and 100 ns respectively), the echo-detected fieldsweep spectrum (EDFS) reveals that the echo intensity spans from $2 \mathrm{G}$ up to $6000 \mathrm{G}$. No spin echo can be observed in the absence of the magnetic field. Nevertheless, a slight static field of $2 \mathrm{G}$ is able to induce the spin echo. This broad field range over which excitations are possible arises from several factors: the $\mathrm{Gd}^{3+}$ ions are highly disordered, leading to strains in the spin Hamiltonian; the orientations of the molecules in the ensemble are homogeneously distributed, leading to a powder spectrum in which the anisotropy energy and the Zeeman energy are comparable; and the microwave pulses of 50 ns duration excite spins over a bandwidth of $20 \mathrm{MHz}$.

The spin echo can be observed below $15 \mathrm{~K}$ in the field range studied here, and we measure the spin-lattice relaxation $\left(T_{1}\right)$ and the quantum phase memory time $\left(T_{m}\right)$ under these conditions. Both $T_{1}$ and $T_{m}$ vary as a function of the magnetic field and the temperature. At $6 \mathrm{~K}, T_{1}$ is determined to be 2.3(1) $\mu$ s in the absence of the field, and increases to 5.8(1) $\mu$ at $3386 \mathrm{G}$, while higher magnetic field reduces $T_{1}$ down to 3.9(2) $\mu$ s (Table 1). This phenomenon is similar to the observation by Sessoli in $S=1 / 2$ mononuclear complexes, where two competing effects of spin-spin/nuclear and spin-phonon interactions are proposed to explain this observation ${ }^{27}$. However, the $T_{m}$ of a transition corresponding to a certain direction is a monotonically-decreasing function of field. This could be due to the fact that, owing to competition between the anisotropy and Zeeman energies, the levels involved in the superposition states are determined by the magnetic field at X-band. In the full field range, $T_{1}$ is reduced by one order of magnitude by increasing the temperature from 5 to $14 \mathrm{~K}$ (Figure 3b), whereas $T_{m}$ only slightly decreases upon warming. The longest $T_{m}$ is found to be $1.60(1) \mu \mathrm{s}$ at 
$5 \mathrm{~K}$ corresponding to the $x$-direction transition at $2513 \mathrm{G}$, which can be further enhanced by dynamical decoupling (DD) to 5.21(12) $\mu$ s (Figure S4.6). It is notable to observe that the $T_{m}$ (1.93(9) $\mu \mathrm{s})$ is observed to increase to around $2 T_{1}(2.26(3) \mu \mathrm{s})$ at $10 \mathrm{~K}$ by DD. The limit of $T_{m}$ occurs when most of the nuclear spin effect is suppressed by $\mathrm{DD}^{28}$.

Table 1 | Relaxation time values and transition information of $\mathbf{G d}_{2} @ \mathrm{C}_{79} \mathrm{~N}$ at $6 \mathrm{~K}$

\begin{tabular}{|c|c|c|c|c|c|c|c|}
\hline$B_{0} / \mathrm{G}$ & $T_{1} / \mu \mathrm{s}$ & $T_{m} / \mu \mathrm{s}$ & *Transition & $B_{0} / \mathrm{G}$ & $T_{1} / \mu \mathrm{s}$ & $T_{m} / \mu \mathrm{s}$ & *Transition \\
\hline \multicolumn{4}{|c|}{$x$ direction } & \multicolumn{4}{|c|}{$z$ direction } \\
\hline 2474 & 4.3(1) & $1.28(2)$ & $\left|-\frac{7}{2}\right| \leftrightarrow\left|-\frac{5}{2}\right|$ & 116.2 & $3.2(1)$ & $0.35(1)$ & $\left|-\frac{9}{2}\right| \leftrightarrow\left|-\frac{11}{2}\right|$ \\
\hline 2513 & 4.4(1) & $1.25(2)$ & $\left|-\frac{5}{2}\right| \leftrightarrow\left|-\frac{3}{2}\right|$ & 590.4 & $2.8(1)$ & $0.31(1)$ & $\left|+\frac{7}{2}\right| \leftrightarrow\left|+\frac{9}{2}\right|$ \\
\hline 2597 & 4.4(1) & $1.25(1)$ & $\left|-\frac{3}{2}\right| \leftrightarrow\left|-\frac{1}{2}\right|$ & 1293 & $3.8(1)$ & $0.23(1)$ & $\left|+\frac{5}{2}\right| \leftrightarrow\left|+\frac{7}{2}\right|$ \\
\hline 2728 & 4.7(1) & $1.24(1)$ & $\left|-\frac{1}{2}\right| \leftrightarrow\left|+\frac{1}{2}\right|$ & 1993 & $4.3(1)$ & $0.98(2)$ & $\left|+\frac{3}{2}\right| \leftrightarrow\left|+\frac{5}{2}\right\rangle$ \\
\hline 2908 & $5.1(1)$ & $1.17(1)$ & $\left|+\frac{1}{2}\right| \leftrightarrow\left|+\frac{3}{2}\right|$ & 2690 & $4.6(1)$ & $1.23(1)$ & $\left|+\frac{1}{2}\right| \leftrightarrow\left|+\frac{3}{2}\right|$ \\
\hline 3142 & $5.3(1)$ & $1.15(1)$ & $\left|+\frac{3}{2}\right| \leftrightarrow\left|+\frac{5}{2}\right|$ & 3386 & $5.8(1)$ & $1.10(1)$ & $\left|-\frac{1}{2}\right| \leftrightarrow\left|+\frac{1}{2}\right|$ \\
\hline \multicolumn{4}{|c|}{$y$ direction } & 4082 & $5.5(1)$ & $0.91(1)$ & $\left|-\frac{3}{2}\right| \leftrightarrow\left|-\frac{1}{2}\right|$ \\
\hline 2788 & $4.8(1)$ & $1.23(1)$ & $\left|-\frac{3}{2}\right| \leftrightarrow\left|-\frac{1}{2}\right|$ & 4781 & $5.2(1)$ & $0.75(1)$ & $\left|-\frac{5}{2}\right| \leftrightarrow\left|-\frac{3}{2}\right|$ \\
\hline 3177 & 5.4(1) & $1.12(1)$ & $\left|-\frac{1}{2}\right| \leftrightarrow\left|+\frac{1}{2}\right|$ & 5484 & $4.0(2)$ & $0.57(2)$ & $\left|-\frac{7}{2}\right| \leftrightarrow\left|-\frac{5}{2}\right|$ \\
\hline 3586 & $5.8(1)$ & $1.02(1)$ & $\left|+\frac{1}{2}\right| \leftrightarrow\left|+\frac{3}{2}\right|$ & \multirow{5}{*}{\multicolumn{4}{|c|}{$\begin{array}{l}\text { * The transitions are assigned according to the high field } \\
\text { approximation for the } x \text { and } y \text { directions. The } z \text { direction } \\
\text { transitions are assigned by ignoring the } E\left(\hat{S}_{x}^{2}-\hat{S}_{y}^{2}\right) \text { term. }\end{array}$}} \\
\hline 4025 & $5.6(1)$ & $0.90(1)$ & $\left|+\frac{3}{2}\right| \leftrightarrow\left|+\frac{5}{2}\right|$ & & & & \\
\hline 4505 & $5.3(1)$ & $0.81(1)$ & $\left|+\frac{5}{2}\right| \leftrightarrow\left|+\frac{7}{2}\right|$ & & & & \\
\hline 5037 & $4.8(1)$ & $0.73(1)$ & $\left|+\frac{7}{2}\right| \leftrightarrow\left|+\frac{9}{2}\right|$ & & & & \\
\hline 5650 & $3.9(2)$ & $0.66(2)$ & $\left|+\frac{9}{2}\right| \leftrightarrow\left|+\frac{11}{2}\right|$ & & & & \\
\hline
\end{tabular}

The quantum coherence behavior of this anisotropic high spin molecule is better than those previously reported ${ }^{21-22,29}$. The long phase memory times allow manipulation of the coherent superpositions between multiple levels in $\mathrm{Gd}_{2} @ \mathrm{C}_{79} \mathrm{~N}$, which is the key ingredient for implementing Grover's algorithm in molecular magnets ${ }^{18}$. This is most simply achieved by employing a sequence of consecutive microwave pulses, each with a different frequency corresponding to a selected EPR transition in $\mathrm{Gd}_{2} @ \mathrm{C}_{79} \mathrm{~N}$. For instance, to populate the system up to a given $\left|M_{S}\right\rangle$ level from the $|-S\rangle$ state $(S=15 / 2)$, a number of $S-M_{S}$ coherent pulses with the frequencies of $\omega_{-S}, \omega_{-S+1}, \cdots, \omega_{M_{S}-1}$ would be applied successively. Here $\omega_{M_{S}}$ corresponds to the EPR transition frequency between the $\left|M_{S}\right\rangle$ and $\left|M_{S}+1\right\rangle$ states, and the durations and phases of the pulses are chosen to obtain the desired superposition in the final state.

The time required for this process is determined by the constraint on the bandwidth of the pulses; for a given pulse shape, the pulse bandwidth is inversely proportional to the pulse duration. The 
bandwidths of the pulses should be significantly less than the difference between any two $\omega_{M_{S}}$ values to avoid any undesired crosstalk between levels. In $\mathrm{Gd}_{2} @ \mathrm{C}_{79} \mathrm{~N}$ at $1.4 \mathrm{~T}$ applied parallel to the $z$ direction, the anisotropy leads to discrete $\omega_{M_{S}}$ values with separations of at least $1.8 \mathrm{GHz}$, so pulses of $10 \mathrm{~ns}$ (i.e. with bandwidths of order $100 \mathrm{MHz}$ ) are sufficiently long. Thus, the microsecond-scale $T_{m}$ times in $\mathrm{Gd}_{2} @ \mathrm{C}_{79} \mathrm{~N}$ are very substantially longer than would be necessary for creating a coherent superposition involving all $\left|M_{S}\right\rangle$ states, for which 15 pulses would be needed.

Diverse Rabi cycles. In order to confirm that coherent manipulations within the multiplet are possible, we performed Rabi cycles at $5 \mathrm{~K}$ with an echo-detected nutation experiment (Figure 4ab). Besides the Rabi oscillations of the electron spin, nuclear Larmor precession can also modulate the electron spin by hyperfine coupling. The nutation pulses are therefore applied in three distinctive microwave powers (attenuating the full power of $300 \mathrm{~W}$ with 28,34 and $40 \mathrm{~dB}$ ) so as to vary the $\mathrm{B}_{1}$ field. This is to distinguish the Rabi oscillations from the Larmor precessions of the nuclei in the static field. The Rabi frequency of a two-level cycle exhibits a linear function to the $\mathrm{B}_{1}$ field, while the Larmor frequency is a linear function of $\mathrm{B}_{0}$.

Fourier transformation of the time domain data from 2 to $6000 \mathrm{G}$ affords frequency domain spectra versus magnetic fields, as seen in Figure 4d. In all of the fields measured, the linear response of the oscillation frequency to the $\mathrm{B}_{1}$ field holds, confirming that the diverse Rabi cycles are detected. It is worth noting that at any field, only one type of Rabi oscillation can be detected, and no multiphoton coherence is observed ${ }^{30}$. For the hint of the nuclear effect on the spectra, weak slants are observable in Figure $4 \mathrm{~d}$ for 34 and $40 \mathrm{~dB}$, which indicates the oscillation frequencies vary upon $\mathrm{B}_{0}$ field. Careful comparison of the two slant reveals identical slopes $(\sim 6.54 \mathrm{MHz} / \mathrm{T})$. This is a strong indication that these signals originate from the Larmor precession of the nuclei, which we assign to deuterium $\left(\frac{\gamma_{D}}{2 \pi}=6.536 \mathrm{MHz} / \mathrm{T}\right)$ from the $d^{8}$-toluene solvent. The Larmor precession signal can be observed over a wide field range (2500 to $5500 \mathrm{G}$ ), implying that the deuterium spins modulate Rabi cycles between various levels of the $S=15 / 2$ multiplet.

Similar to the EDFS experiment, the intensities of the Rabi frequencies are observable below $6000 \mathrm{G}$ in the nutation experiment when $28 \mathrm{~dB}$ attenuated microwave power is applied. This is again principally due to the disorder of the $\mathrm{Gd}^{3+}$ ions and fullerene orientation, as discussed previously. By improving the selectivity of the pulses, it is observable that the intensities of the Rabi frequencies between 1000 and $2000 \mathrm{G}$ are largely suppressed below the noise. The most intense Rabi frequencies are found in the range of 2000 to $4000 \mathrm{G}$, however these correspond to different transitions and are not discrete in the field domain for the same reasons discussed above.

It is notable that the Rabi frequencies vary depending on the static field, as, at a fixed microwave frequency, $\mathrm{B}_{0}$ works as a controller to select the distinctive transitions of the $S=15 / 2$ multiplet. The cw-EPR spectra reveal that it is necessary to describe the molecule with a rhombic symmetry. Consequently, the Hamiltonian of the system does not commute with the operator $\hat{S}_{z}$, so $M_{s}$ are not well-defined quantum numbers. It is therefore not possible to apply the well-known formula $\left(\Omega_{R}^{M_{S} \leftrightarrow M_{S+1}}=g \mu_{B} B_{1} \sqrt{S(S+1)-M_{S}\left(M_{S}+1\right)}\right)$ to calculate Rabi frequencies between two specific energy levels ${ }^{28}$. Nevertheless, based on the unitary transformation and rotating-wave 
approximation approach (see SI 4), we calculated these frequencies with the Hamiltonian parameters determined by cw-EPR spectra. Only the Rabi frequencies of the transitions, which happen in the field direction along the principal axes of the second rank ZFS tensor, are calculated herein. The calculated results are plotted in red dots in Figure 4d, which are consistent with experimental data. This confirms the validation of the cw-EPR simulation and the Rabi frequency calculation method.

As discussed above, it is necessary to put the spin in arbitrary superposition of states so as to implement Grover's algorithm in this high spin molecule. Observation of the diverse Rabi cycles illustrates that the coherent superposition manipulation is achievable and all necessary unitary operations can be performed.

a)

b)
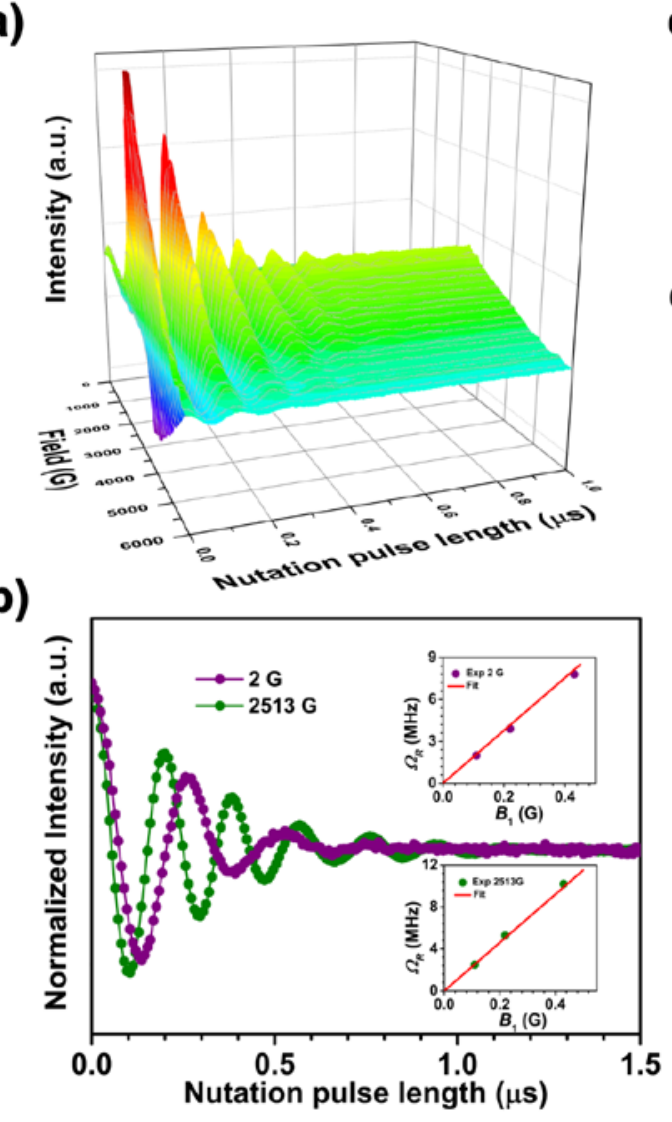

c)

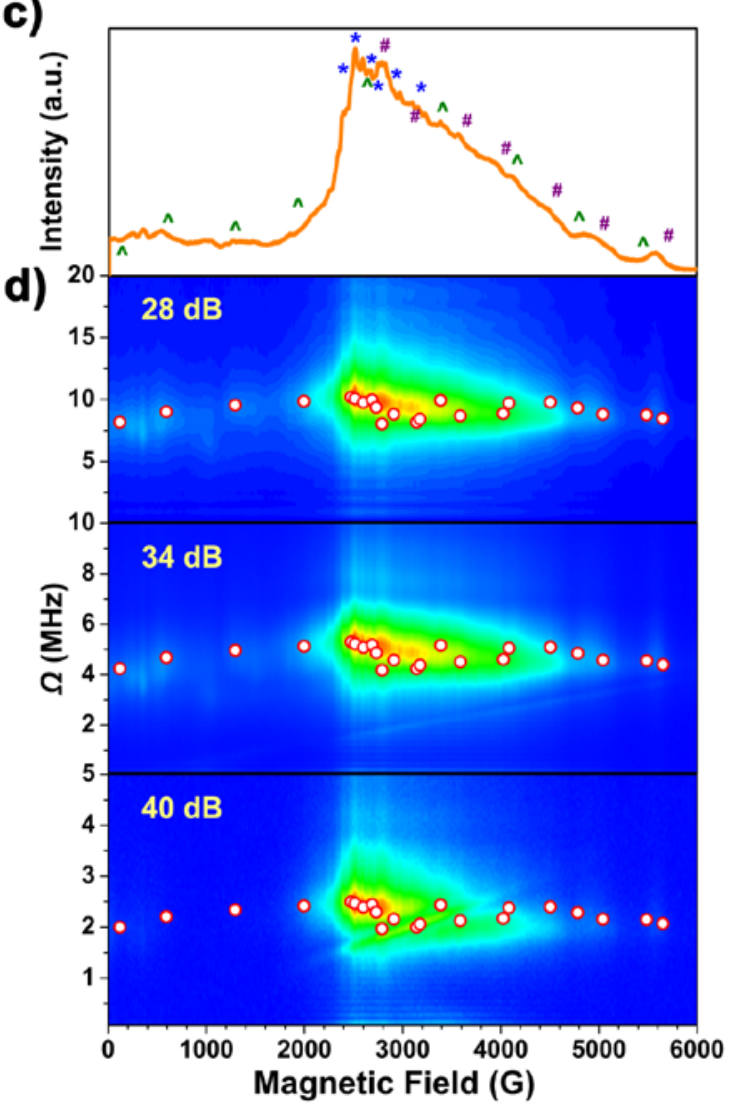

Figure 4 | Rabi oscillations determined in the field range of 2 to $6000 \mathrm{G}$ at $5 \mathrm{~K}$. a) The 3dimensional surface of Rabi oscillations in the field range of 2 to $6000 \mathrm{G}$ at $28 \mathrm{~dB}$ microwave attenuation. b) The oscillations at $2 \mathrm{G}$ (green) and $2513 \mathrm{G}$ (purple) as examples. The linear function of both oscillation frequencies with respect to the $B_{1}$ field are plotted in the insets. The difference of the slops (18.9 MHz/G for $2 \mathrm{G}$ and $23.3 \mathrm{MHz} / \mathrm{G}$ for $2513 \mathrm{G}$ ) of the two linear functions originate from the different transitions. c) X-band echo-detected field-sweep spectrum marked with the transition information. The symbols “*”, “\#” and “^” denote the transitions along $\mathrm{x}, \mathrm{y}$ and $\mathrm{z}$ directions, respectively. d) The three blocks show the FT of the Rabi oscillation at three $\mathrm{B}_{1}$ fields controlled by attenuating the $300 \mathrm{~W}$ microwave power with $28 \mathrm{~dB}, 34 \mathrm{~dB}$ and $40 \mathrm{~dB}$. The open red circles are the calculated Rabi frequencies in the rotating-wave approximation. 


\section{EXPERIMENTAL METHODS}

Synthesis and isolation. $\mathrm{Gd}_{2} @ \mathrm{C}_{79} \mathrm{~N}$ was produced by using a modified arc-discharge method. Briefly, the anode graphite rod filled with $\mathrm{GdNi}_{2} / \mathrm{Fe}_{x} \mathrm{~N}$ ( $x=2-4$ )/graphite powder (atomic ratio=1:0.08:10) was evaporated at $100 \mathrm{~A}$ under 150 torr helium static atmosphere. The soot was then refluxed in o-dichlorobenzene under nitrogen atmosphere for 5 h. $\mathrm{Gd}_{2} @ \mathrm{C}_{79} \mathrm{~N}$ was isolated via a multi-step HPLC procedure and checked by laser desorption time-of-flight (LD-TOF) mass spectroscopy.

Single-crystal X-ray diffraction. A single black co-crystal of $\mathrm{Gd}_{2} @ \mathrm{C}_{79} \mathrm{~N} / \mathrm{Ni}^{\mathrm{II}}(\mathrm{OEP})$ was obtained by allowing the benzene solution of $\mathrm{Gd}_{2} @ \mathrm{C}_{79} \mathrm{~N}$ and the chloroform solution of $\mathrm{Ni}^{\mathrm{II}}(\mathrm{OEP})$ to diffuse together. X-ray data were collected at $90 \mathrm{~K}$ using a Rigaku Oxford diffractometer equipped with a CCD collector. Multi-scan method was used for absorption correction. The structure was resolved using direct methods (SHELXS97) and refined on $\mathrm{F}^{2}$ using full-matrix least squares using SHELXL97 ${ }^{31}$. The intact cage was modeled via the crystallographic mirror plane in refinement. This data can be obtained free of charge from The Cambridge Crystallographic Data Centre with CCDC Nos. 1577180.

Magnetic measurements. The powder sample of $\mathrm{Gd}_{2} @ \mathrm{C}_{79} \mathrm{~N}$ for dc magnetic measurements were wrapped in $\mathrm{Al}$ foil and fixed on the straw with slight amount of N-grease. Dc experiments were performed on a Quantum Design MPMS XL-5 SQUID magnetometer. Magnetic data were corrected for the paramagnetism from the $\mathrm{Al}$ foil and the diamagnetic contribution of the sample calculated from Pascal's constants. The magnetization and magnetic susceptibility data are simulated by EasySpin toolbox ${ }^{32}$ (http://www.easyspin.org/) based on Matlab.

EPR measurements. The $\mathrm{Gd}_{2} @ \mathrm{C}_{79} \mathrm{~N}$ was dissolved in a $d^{8}$-toluene solution for EPR experiments with the spin concentration of $5.3 \times 10^{13}$ per milliliter determined by the spin counting method. CWEPR spectra were measured on a Bruker Elexsys E580 spectrometer with a Super-high sensitivity probe head $(\omega=9.36 \mathrm{GHz})$. Pulsed EPR data were collected on the same system by MS-3 cavity $(\omega=9.33 \mathrm{GHz})$. The pulsed Q-band measurements were performed in Oxford-CAESR on a Bruker Elexsys E580 with a EN 5107D2 Q-Band EPR/ENDOR Probehead $2 \mathrm{~mm}$ sample diameter cavity $(\omega=33.89 \mathrm{GHz})$. The cw-EPR spectra are simulated by EasySpin toolbox ${ }^{32}$ (http://www.easyspin.org/) based on Matlab. The low-temperature environment was achieved by Oxford Instruments produced liquid helium cryostats (ESR900 for cw and CF935 for pulse). The signal of the pulsed-EPR experiments was collected by integrating the Hahn echo ( $\pi / 2-\tau-\pi$ - $\tau$-echo) with $\tau=200 \mathrm{~ns}$. The $T_{1}$ values were measured by the inversion recovery method $(\pi-\mathrm{T}-\pi / 2-\tau-\pi-\tau-$ echo) with 4-step phase cycling. The $T_{m}$ values were obtained by increasing the $\tau$ value of Hahn echo sequence with 4-step phase cycling. The dynamic decoupling measurements were carried out by CPMG sequence $\left(\pi_{\mathrm{x}} / 2-\tau-\left(\pi_{\mathrm{y}}-2 \tau\right)_{\mathrm{n}}-\pi_{\mathrm{y}}-\tau\right.$-echo) with 16 -step phase cycling. The $\pi / 2$ and $\pi$ pulse lengths in EDFS, $T_{1}$ and $T_{m}$ measurements were 50 and 100 ns with $34 \mathrm{~dB}$ attenuation of the microwave power $300 \mathrm{~W}$, respectively. The nutation experiments were carried out with standard sequence ( $t_{p}-\mathrm{T}-\pi / 2-\tau-\pi$ - $\tau$-echo), where $\mathrm{T}>5 T_{m}$. The $\pi / 2$ pulse lengths were adjusted to 26,50 and 100 ns by $28 \mathrm{~dB}, 34 \mathrm{~dB}$ and $40 \mathrm{~dB}$ attenuation. 


\section{CONCLUSION AND OUTLOOK}

In summary, the crystallographic studies of dimetallic azafullerene $\mathrm{Gd}_{2} @ \mathrm{C}_{79} \mathrm{~N}$ reveals its $C_{s}$ or lower molecular symmetry. Well-defined high spin ground state with long quantum coherence time and diverse Rabi cycles are further discovered, demonstrating that the anisotropic EMF Gd $\mathrm{d}_{2} @ \mathrm{C}_{79} \mathrm{~N}$ fulfills the requirements for implementing Grover's algorithm in molecular magnet. This research verifies the merits of utilizing EMFs as spin qubit candidates through rational design. In principle, selection of $\mathrm{Gd}^{3+}$ as the major spin contributor results in slight anisotropy ${ }^{29}$, affording nonequidistant energy levels. Other $4 \mathrm{f}$-elements would lead to very large anisotropy and could decohere the quantum phase rapidly. To gain a larger spin, we include a second $\mathrm{Gd}^{3+}$ ion in the molecule. However, the magnetic coupling between rare-earth ions is normally smaller than $1 \mathrm{~cm}^{-1}$. The superposition states would collapse due to the flip-flop effect of adjacent paramagnetic centers. We overcome this by introducing an unpaired electron in the system, which often couples with 4felectrons very well ${ }^{33}$. The strong coupling makes the three spin carriers exhibit a total spin of $S=$ $15 / 2$. Nevertheless, high spin molecules often behave more classically, coupling to the environment efficiently resulting in a short phase memory time ${ }^{34-35}$. The barren nuclear-spin "Faraday cage" is therefore employed to protect the giant spin; azafullerene therefore works as a shelter and a free electron donor simultaneously. In our case, the single spin unit with magnetic moment of $14.7 \mu_{B}$ and extremely large coupling constant $\left(J_{G d-R a d}=350 \pm 20 \mathrm{~cm}^{-1}\right)$ has been obtained through this strategy, further corroborating the charge transfer mechanism in this system. The as-prepared $\mathrm{Gd}_{2} @ \mathrm{C}_{79} \mathrm{~N}$ exhibits long coherence time for each superposition state for quantum manipulation. More importantly, the anisotropy offers us a window to view the quantum coherence between various energy levels, rendering $\mathrm{Gd}_{2} @ \mathrm{C}_{79} \mathrm{~N}$ as the first EMF qubit exhibiting diverse Rabi cycles controlled by the magnetic field, demonstrating that arbitrary superposition states can be realized between diverse levels. The present research is based on molecular ensemble. For the purpose of quantum information processing, individual molecular devices are necessary. The EMF structure is maneuverable, offering the possibility of application in molecular spintronics. ${ }^{36}$

\section{AUTHOR INFORMATION}

\section{Corresponding Authors}

*zjshi@pku.edu.cn

*jiangsd@pku.edu.cn

*gaosong@pku.edu.cn

\section{Author Contributions}

${ }^{\dagger}$ Z.H. and B.-W.D. contributed equally to this work.

\section{Notes}

The authors declare no competing financial interest.

\section{ACKNOWLEDGEMENTS}

This work was supported by the National Natural Science Foundation of China (grant nos. 21471010, 21621061, 21601005), the National Basic Research Program of China (grant nos. 2013CB933401, 
2013CB933402 and 2017YFA024901) and the Young Elite Scientist Sponsorship Program of the China Association of Science and Technology (grant no. YESS20150011). We would like to thank Dr. Si-Xue Qin, Dr. Ya Wang and Prof. Jun-Liang Sun for scientific discussion. We would also like to thank Prof. Zhe-Ming Wang for assistance in magnetic experiments.

\section{REFERENCES}

1. Popov, A. A.; Yang, S.; Dunsch, L., Chem. Rev. 2013, 113 (8), 5989-6113.

2. Westerstrom, R.; Dreiser, J.; Piamonteze, C.; Muntwiler, M.; Weyeneth, S.; Brune, H.; Rusponi, S.; Nolting, F.; Popov, A.; Yang, S.; Dunsch, L.; Greber, T., J. Am. Chem. Soc. 2012, 134 (24), 98409843.

3. Liu, F.; Gao, C. L.; Deng, Q.; Zhu, X.; Kostanyan, A.; Westerstrom, R.; Wang, S.; Tan, Y. Z.; Tao, J.; Xie, S. Y.; Popov, A. A.; Greber, T.; Yang, S., J. Am. Chem. Soc. 2016, 138 (44), 14764-14771.

4. Liu, F.; Krylov, D. S.; Spree, L.; Avdoshenko, S. M.; Samoylova, N. A.; Rosenkranz, M.; Kostanyan, A.; Greber, T.; Wolter, A. U. B.; Buchner, B.; Popov, A. A., Nat. Commun. 2017, 8, 16098.

5. Liu, F.; Wang, S.; Gao, C. L.; Deng, Q.; Zhu, X.; Kostanyan, A.; Westerström, R.; Jin, F.; Xie, S. Y.; Popov, A. A., Angew. Chem. 2017, 129 (7), 1856-1860.

6. Shinohara, H.; Sato, H.; Ohkohchi, M.; Ando, Y.; Kodama, T.; Shida, T.; Kato, T.; Saito, Y., Nature 1992, 357 (6373), 52-54.

7. Zuo, T.; Xu, L.; Beavers, C. M.; Olmstead, M. M.; Fu, W.; Crawford, T. D.; Balch, A. L.; Dorn, H. C., J. Am. Chem. Soc. 2008, 130 (39), 12992-7.

8. Kurihara, H.; Iiduka, Y.; Rubin, Y.; Waelchli, M.; Mizorogi, N.; Slanina, Z.; Tsuchiya, T.; Nagase, S.; Akasaka, T., J. Am. Chem. Soc. 2012, 134 (9), 4092-5.

9. Wang, T.; Wu, J.; Xu, W.; Xiang, J.; Lu, X.; Li, B.; Jiang, L.; Shu, C.; Wang, C., Angew. Chem. Int. Ed. Engl. 2010, 49 (10), 1786-9.

10. Wu, B.; Wang, T.; Feng, Y.; Zhang, Z.; Jiang, L.; Wang, C., Nat. Commun. 2015, 6, 6468.

11. Ma, Y.; Wang, T.; Wu, J.; Feng, Y.; Jiang, L.; Shu, C.; Wang, C., Chem. Commun. (Camb) 2012, 48 (94), 11570-2.

12. Feng, Y.; Wang, T.; Li, Y.; Li, J.; Wu, J.; Wu, B.; Jiang, L.; Wang, C., J. Am. Chem. Soc. 2015, 137 (47), 15055-60.

13. Feynman, R. P., Int. J. Theor. Phys. 1982, 21 (6-7), 467-488.

14. Shor, P. W. In Algorithms for quantum computation: discrete logarithms and factoring, Proceedings 35th Annual Symposium on Foundations of Computer Science, 20-22 Nov 1994; 1994; pp 124-134.

15. Grover, L. K., Phys. Rev. Lett. 1997, 79 (23), 4709-4712.

16. Farhi, E.; Gutmann, S., Phys.Rev. A 1998, 57 (4), 2403.

17. Godfrin, C.; Ferhat, A.; Ballou, R.; Klyatskaya, S.; Ruben, M.; Wernsdorfer, W.; Balestro, F., Phys. Rev. Lett. 2017, 119 (18).

18. Leuenberger, M. N.; Loss, D., Nature 2001, 410 (6830), 789-793.

19. Baibekov, E. I.; Gafurov, M. R.; Zverev, D. G.; Kurkin, I. N.; Rodionov, A. A.; Malkin, B. Z.; Barbara, B., Phys. Rev. B 2017, 95 (6), 064427.

20. Jenkins, M. D.; Duan, Y.; Diosdado, B.; Garcia-Ripoll, J. J.; Gaita-Arino, A.; Gimenez-Saiz, C.; Alonso, P. J.; Coronado, E.; Luis, F., Phys. Rev. B 2017, 95 (6), 064423.

21. Schlegel, C.; van Slageren, J.; Manoli, M.; Brechin, E. K.; Dressel, M., Phys. Rev. Lett. 2008, 101 (14), 147203. 
22. Takahashi, S.; Tupitsyn, I. S.; van Tol, J.; Beedle, C. C.; Hendrickson, D. N.; Stamp, P. C. E., Nature 2011, 476 (7358), 76-79.

23. Fu, W.; Zhang, J.; Fuhrer, T.; Champion, H.; Furukawa, K.; Kato, T.; Mahaney, J. E.; Burke, B. G.; Williams, K. A.; Walker, K.; Dixon, C.; Ge, J.; Shu, C.; Harich, K.; Dorn, H. C., J. Am. Chem. Soc. 2011, 133 (25), 9741-50.

24. Singh, M. K.; Yadav, N.; Rajaraman, G., Chem. Commun. (Camb) 2015, 51 (100), 17732-5.

25. Stevenson, S.; Phillips, J. P.; Reid, J. E.; Olmstead, M. M.; Rath, S. P.; Balch, A. L., Chem. Commun. (Camb) 2004, (24), 2814-5.

26. Furukawa, K.; Okubo, S.; Kato, H.; Shinohara, H.; Kato, T., J. Phys. Chem. A 2003, 107 (50), 10933-10937.

27. Atzori, M.; Tesi, L.; Morra, E.; Chiesa, M.; Sorace, L.; Sessoli, R., J. Am. Chem. Soc. 2016, 138 (7), 2154-2157.

28. Schweiger, A.; Jeschke, G., Principles of Pulse Electron Paramagnetic Resonance. Oxford University Press: 2001.

29. Martinez-Perez, M. J.; Cardona-Serra, S.; Schlegel, C.; Moro, F.; Alonso, P. J.; Prima-Garcia, H.; Clemente-Juan, J. M.; Evangelisti, M.; Gaita-Arino, A.; Sese, J.; van Slageren, J.; Coronado, E.; Luis, F., Phys. Rev. Lett. 2012, 108 (24), 247213.

30. Bertaina, S.; Chen, L.; Groll, N.; Van Tol, J.; Dalal, N. S.; Chiorescu, I., Phys. Rev. Let.t 2009, 102 (5), 050501.

31. Sheldrick, G. M., Acta Crystallogr. Sect. A: Found. Crystallogr. 2008, 64 (1), 112-122.

32. Stoll, S.; Schweiger, A., J. Magn. Reson. 2006, 178 (1), 42-55.

33. Rinehart, J. D.; Fang, M.; Evans, W. J.; Long, J. R., Nat. Chem. 2011, 3 (7), 538-542.

34. Graham, M. J.; Zadrozny, J. M.; Shiddiq, M.; Anderson, J. S.; Fataftah, M. S.; Hill, S.; Freedman, D. E., J. Am. Chem. Soc. 2014, 136 (21), 7623-7626.

35. Morello, A.; Stamp, P. C. E.; Tupitsyn, I. S., Phys. Rev. Lett. 2006, 97 (20), 207206.

36. Bogani, L.; Wernsdorfer, W., Nat. Mater. 2008, 7 (3), 179-186. 


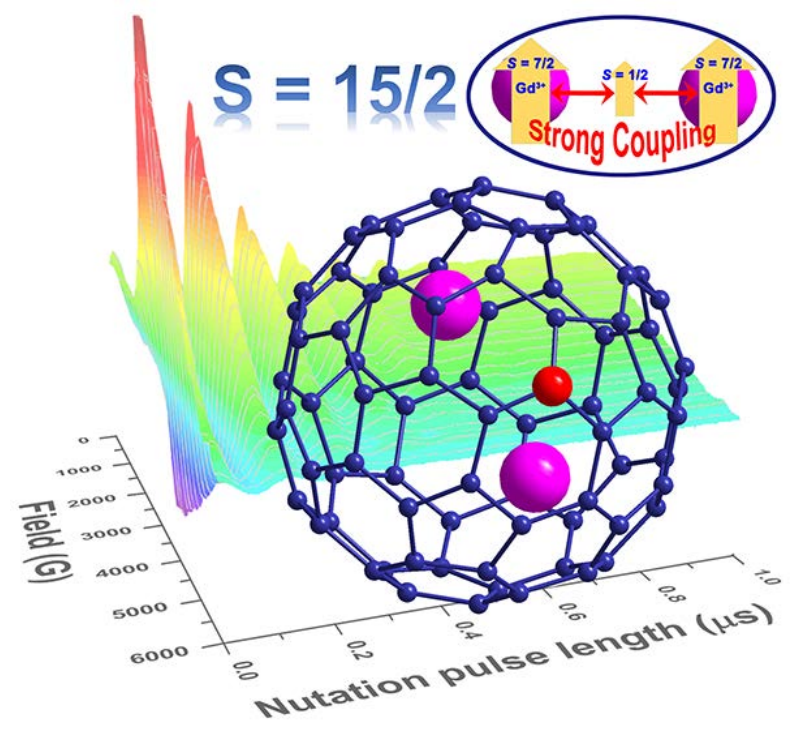

